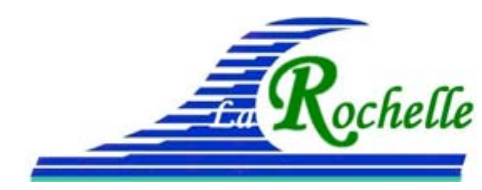

XVèmes Journées Nationales Génie Côtier - Génie Civil

La Rochelle, 29 au 31 mai 2018

DOI:10.5150/jngcgc.2018.012 (C) Editions Paralia CFL

disponible en ligne - http://www.paralia.fr - available online

\title{
Modélisation de l'interaction de la houle en théorie des ondes longues lors de sa propagation sur un fond poreux stratifié
}

\author{
Mustapha MOUHID ${ }^{1}$, Mohamed CHAGDALI ${ }^{1}$
}

\author{
1. Université Hassan II Casablanca, Faculté des Sciences Ben M'Sik, LPPPC, \\ Av Driss El Harti, BP 7556, Sidi Othman, Casablanca, Maroc. \\ mouhidmustapha@gmail.com; chagdalimohamed@gmail.com
}

\section{Résumé :}

On présente une modélisation analytique d'un écoulement engendré par la houle dans un milieu poreux constitué de trois couches stratifiées et de caractéristiques géométriques et mécaniques différentes. La formulation mathématique étudiée est basée sur la théorie potentielle de la houle avec l'approximation des ondes longues. La procédure du calcul analytique développée dans ce travail, se compose de trois étapes :

- Subdivision du domaine d'étude en plusieurs sous-domaines. Le domaine externe est le lieu de propagation de la houle. Les autres sous-domaines présentent les trois couches du milieu poreux.

- Résolution analytique dans chaque sous-domaine. Dans le domaine externe, la forme de la solution générale est obtenue par la résolution du problème de la houle au premier ordre en approximation des ondes longues. Dans le milieu poreux, la théorie de SOLLITT et CROSS (1972) va servir comme base pour modéliser la forme de la solution générale dans le milieu poreux.

- Dans la troisième étape, on raccorde les solutions entre l'ensemble des sous-domaines par la continuité des flux et des pressions.

Par cette procédure, on calcule la relation de dispersion des paramètres de la houle en présence d'un fond poreux multicouche dans le cadre de théorie des ondes longues ainsi que la cinématique de l'écoulement engendré par la houle dans le milieu poreux. Plus particulièrement, on s'intéresse à la profondeur de fermeture de l'impact de la houle dans le milieu poreux. L'analyse sera faite en fonction de deux paramètres: La porosité et l'épaisseur des couches stratifiées.

\section{Mots-clés :}

Interaction houle milieux poreux, Formulation analytique, Théorie des ondes longues, Profondeur de fermeture.

\section{Introduction}

Lors de la propagation de la houle sur des fonds marins considérés comme des milieux poreux; la perméabilité de ces milieux induit un écoulement interne et des flux non négligeables au travers de l'interface milieu poreux-milieu externe. Ce type de problème a fait l'objet de plusieurs recherches en génie côtier. La théorie de SOLITT \& CROSS 


\section{Thème 1 - Hydrodynamique côtière}

(1972) a servi comme base théorique pour modéliser divers problèmes dans ce domaine. L'approche a été également appliquée à l'analyse des ondes longues par MADSEN (1983). LEE et LAN (1995) a développé une méthode généralisée pour des milieux poreux avec des couches multiples et des régions multiples. BROSSARD et al., (2004) ont proposé, par une approche analytique, validée expérimentalement dans un canal à houle, la relation de dispersion de l'écoulement dans un milieu poreux mono disperse et rigide.

Dans ce travail, on généralise la relation de dispersion à un milieu poreux constitué de plusieurs couches stratifiées dans le cadre de l'hypothèse de la propagation des ondes longues. Le modèle théorique utilisé est celui de Forchheimer. Ce modèle est adapté aux écoulements instationnaires et il permet de tenir compte des forces de frottement et des termes d'inertie. Nous procédons par une approche analytique linéaire au premier ordre en adoptant le principe de Lorentz, (SOLITT \& CROSS, 1972).

\section{Formulation théorique}

Nous supposons que le problème est plan et que la hauteur externe est $h_{e}$. Chaque couche possède une épaisseur $h_{i}$ pour $\mathrm{i}=1,2$ et 3 .

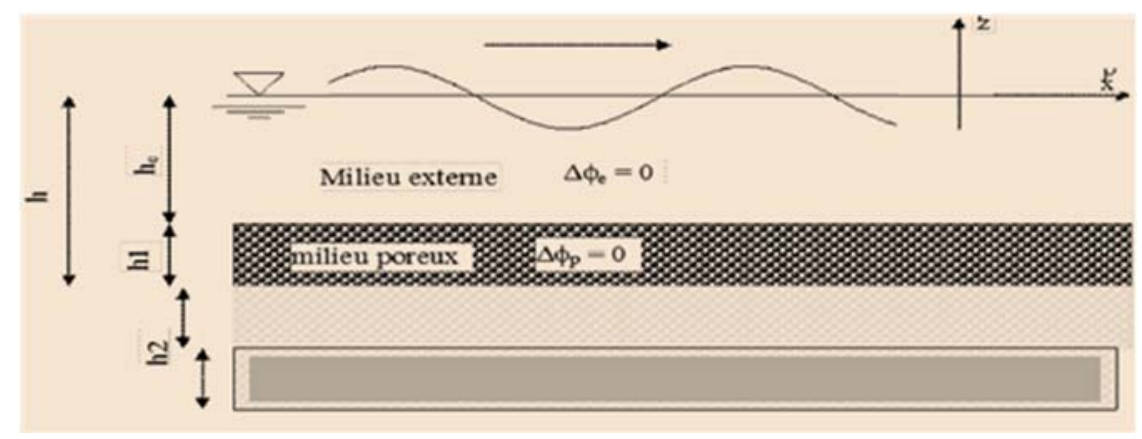

Figure 1. Schémas d'étude.

Le problème de la propagation de la houle, en présence d'un fond poreux, est défini dans le cadre potentiel. Le fluide est parfait et incompressible. L'écoulement est plan et irrotationnel. En homogénéisant le milieu poreux on peut introduire aussi un potentiel de vitesse. Dans les deux milieux, on aura à résoudre un problème à potentiel des vitesses SOLLITT et CROSS (1979) et BROSSARD et al, (2004).

Dans la suite de ce travail, on utilise les notations suivantes pour formuler les équations dans un repère cartésien dont l'origine est sur la ligne moyenne de la surface libre du canal.

$\phi_{j}$ : $\quad$ Potentiel des vitesses dans la couche d'épaisseur j ;

$\phi_{e}$ : Potentiel des vitesses dans la couche externe (domaine de la houle);

$\eta$ : L'élévation de la surface libre par rapport à la ligne moyenne $\mathrm{z}=0$;

G : L'accélération de la pesanteur 


\section{XVèmes Journées Nationales Génie Côtier - Génie Civil \\ La Rochelle, 29 au 31 mai 2018}

$\mathrm{t}: \quad$ Le temps ;

$\varepsilon: \quad$ La porosité ;

$\mathrm{d}: \quad$ Le diamètre des particules,

$\alpha: \quad$ Coefficient de tortuosité,

$K$ : La perméabilité intrinsèque définie par l'expression :

$K=\frac{\varepsilon^{2} d^{2}}{\alpha(1-\varepsilon)^{3}}$

$\vartheta$ : $\quad$ La viscosité cinématique,

$C_{f j}: \quad$ Le coefficient de turbulence,

$\mathrm{C}_{\mathrm{f}}$ : Coefficient de frottement,

S: $\quad$ Coefficient de masse ajoutée associé aux particules constituant le milieu poreux granulaire

$s=\frac{1}{\varepsilon}+\frac{1-n}{\varepsilon^{2}} C_{m}$

$f_{j}$ : Le coefficient de dissipation défini par le principe de Lorentz du travail équivalent dissipé dans le milieu poreux

Les équations du mouvement, constituent un problème aux limites qui peuvent être exprimées, en terme de potentiel par:

$$
\begin{aligned}
& \nabla^{2} \Phi_{j}=0 \quad-\infty \leq x \leq+\infty \quad-h_{j} \leq z \leq-h_{j-1} \\
& \frac{\partial \phi_{\mathrm{e}}}{\partial \mathrm{t}}+\mathrm{g} \eta=0 \quad \mathrm{z}=0 \\
& \frac{\partial \phi_{\mathrm{e}}}{\partial \mathrm{z}}=\frac{\partial \eta}{\partial \mathrm{t}} \quad \mathrm{z}=0 \\
& \varepsilon_{j-1} \frac{\partial \Phi_{j-1}}{\partial z}=\varepsilon_{j} \frac{\partial \Phi_{j}}{\partial z} \quad-\infty \leq x \leq+\infty \quad z=h_{j-1} \\
& s_{j-1} \frac{\emptyset_{j-1}}{\partial t}+\omega f_{j-1} \emptyset_{j-1}=s_{j} \frac{\emptyset_{j}}{\partial t}+\omega f_{j} \emptyset_{j} \\
& \frac{\partial \Phi_{n}}{\partial z}=0 \quad z=-h_{n} \\
& f_{j}=\frac{1}{\omega}\left\{\frac{v}{K_{p j}}+\frac{\varepsilon_{j} C_{f j}}{\sqrt{K_{p j}}} \frac{\int_{\Omega}^{t+T} \int_{1}^{t+T}\left|V_{j}\right|^{3} d t d \Omega}{\left|V_{j}\right|^{2} d t d \Omega}\right\}
\end{aligned}
$$

\section{Résolution analytique}

La procédure du calcul analytique développée dans ce travail, se compose de trois étapes: 


\section{Thème 1 - Hydrodynamique côtière}

- Subdivision du domaine d'étude en plusieurs sous-domaines. Le domaine externe est le lieu de propagation de la houle. Les autres sous-domaines présentent les trois couches du milieu poreux, (figure 1).

-Résolution analytique dans chaque sous-domaine. Dans le domaine externe, la forme de la solution générale est obtenue par la résolution du problème de la houle au premier ordre en approximation des ondes longues. Dans le milieu poreux, la théorie de SOLLITT et CROSS (1972) va servir comme base pour modéliser la forme de la solution générale dans le milieu poreux.

- Dans la troisième étape, on raccorde les solutions entre l'ensemble des sous domaines par la continuité des flux et des pressions.

Par cette procédure, on calcule la relation de dispersion des paramètres de la houle en présence d'un fond poreux multicouche dans le cadre de théorie des ondes longues ainsi que la cinématique de l'écoulement engendré par la houle dans le milieu poreux.

L'hypothèse des ondes longues est valable dans le cas où $\mathrm{kh}<<1$. Ce cas se produit en eau peu profonde pour un rapport de kh inférieur à 0,15 (TEMPERVILLE, 1985).

\subsection{Relation de dispersion pour un milieu uni-couche}

Dans le cas où le milieu poreux est constitué d'une seule couche poreuse et rigide, le nombre d'onde $\mathrm{k}$ vérifie l'équation de dispersion :

$$
\omega^{2}=\frac{(f+i s) g k \tanh \left(k h_{e}\right)+i g k \tanh \left(k h_{p}\right)}{(f+i s)+i \tanh \left(k h_{e}\right) \tanh \left(k h_{p}\right)}
$$

La forme de la surface libre est :

$$
\eta(x, t)=a e^{k_{a} x} e^{i\left(\omega t-k_{p} x\right)}
$$

3.2 Relation de dispersion pour un milieu bicouche

Le nombre d'onde $\mathrm{k}$ vérifie l'équation de dispersion

$$
\frac{\omega^{2}}{K g}=\frac{Z_{1}\left(1+Z_{1} t_{0} t_{10}\right) t_{21} / Z_{2}+\left(Z_{1} t_{0}+t_{10}\right)}{Z_{1}\left(Z_{1} t_{10}+t_{0}\right) t_{21} / Z_{2}+\left(Z_{1}+t_{0} t_{10}\right)}
$$

avec: $\quad t_{o}=\operatorname{th}\left(K h_{0}\right) ; t_{21}=\operatorname{th}\left(K\left(h_{2}-h_{1}\right)\right)$ et $Z_{j}=f_{j}+i s_{j}$

On note que dans le cas d'un milieu monocouche qui correspond aux paramètres $t_{21}=$ 0 et $h_{2}=h_{1}$, on retrouve la relation de dispersion (10).

$\frac{\omega^{2}}{K g}=\frac{Z_{1} t_{0}+t_{10}}{Z_{1}+t_{0} t_{10}}$

En absence du milieu poreux, on retrouve la relation de dispersion en classique de la houle linéaire.

$$
\frac{\omega^{2}}{K g}=t_{0}=t h\left(K h_{0}\right)
$$




\section{XVèmes Journées Nationales Génie Côtier - Génie Civil \\ La Rochelle, 29 au 31 mai 2018}

\subsection{Approximation des ondes longues pour un milieu multicouche}

Dans le cas des ondes longues d'onde ( $\mathrm{kh}<<1$ ), (TEMPERVILLE, 1985), en utilisant un développement limité au voisinage du terme Kho et en négligeant les ordres supérieurs à un, la relation de dispersion d'un multi couche peut se mettre sous une forme homogène:

$$
\frac{\omega^{2}}{K g} \approx t h K H \quad H=\frac{h_{0}}{Z_{0}}+\frac{h_{10}}{Z_{1}}+\frac{h_{21}}{Z_{2}}+\frac{h_{32}}{Z_{3}}+\ldots \ldots+\frac{h_{n n-1}}{Z_{n}}
$$

\subsection{Notion du milieu équivalent}

On retrouve la relation de dispersion habituelle avec un milieu équivalent (milieu externe+milieu poreux) de hauteur H. On remarque que si les épaisseurs des couches poreuses et celle du milieu externe sont identiques à une épaisseur e, le multicouche est équivalent à un mono couche d'épaisseur équivalent he et d'impédance équivalente $\mathrm{Z}_{\mathrm{e}}$ tels que :

$$
h_{e}=\frac{e}{Z_{e}} \quad H=h_{0}+h \quad \frac{1}{Z_{e}}=\sum_{i=1}^{n} \frac{1}{Z_{i}} \quad Z_{e}=s_{e}-i f_{e}
$$

\subsection{Notion de la profondeur de fermeture}

Le calcul analytique du potentiel des vitesses permet de définir la profondeur de fermeture, c'est-à-dire la profondeur limite de remobilisation des sédiments dans le milieu poreux. Ce résultat est illustré sur la figure 2 . 
Thème 1 - Hydrodynamique côtière

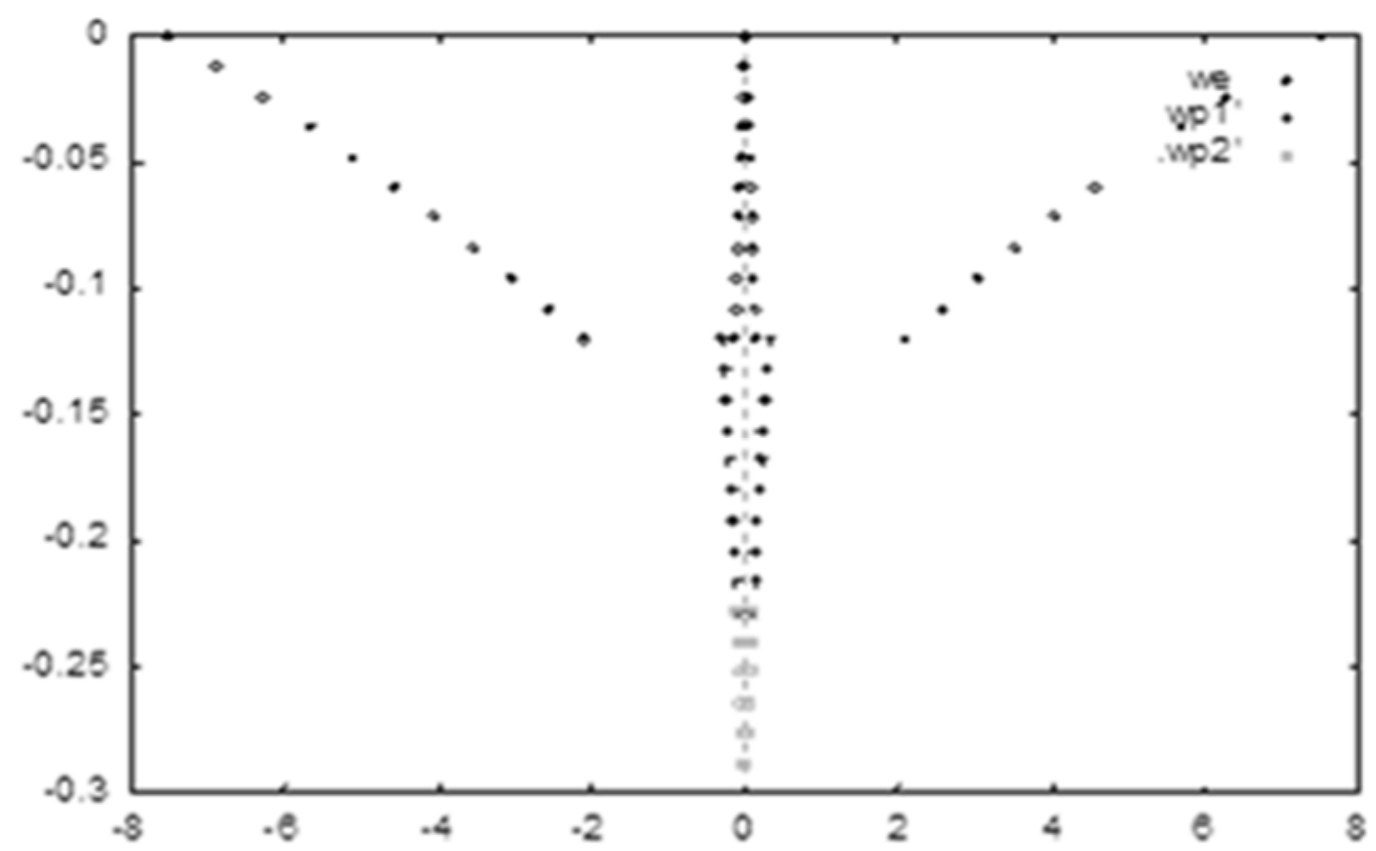

Figure 2. Profil de la composante vertical de la vitesse.

Sur la figure 3, nous présentons l'évolution de la surface libre en présence d'un milieu poreux composé de deux couches de caractéristiques différentes.

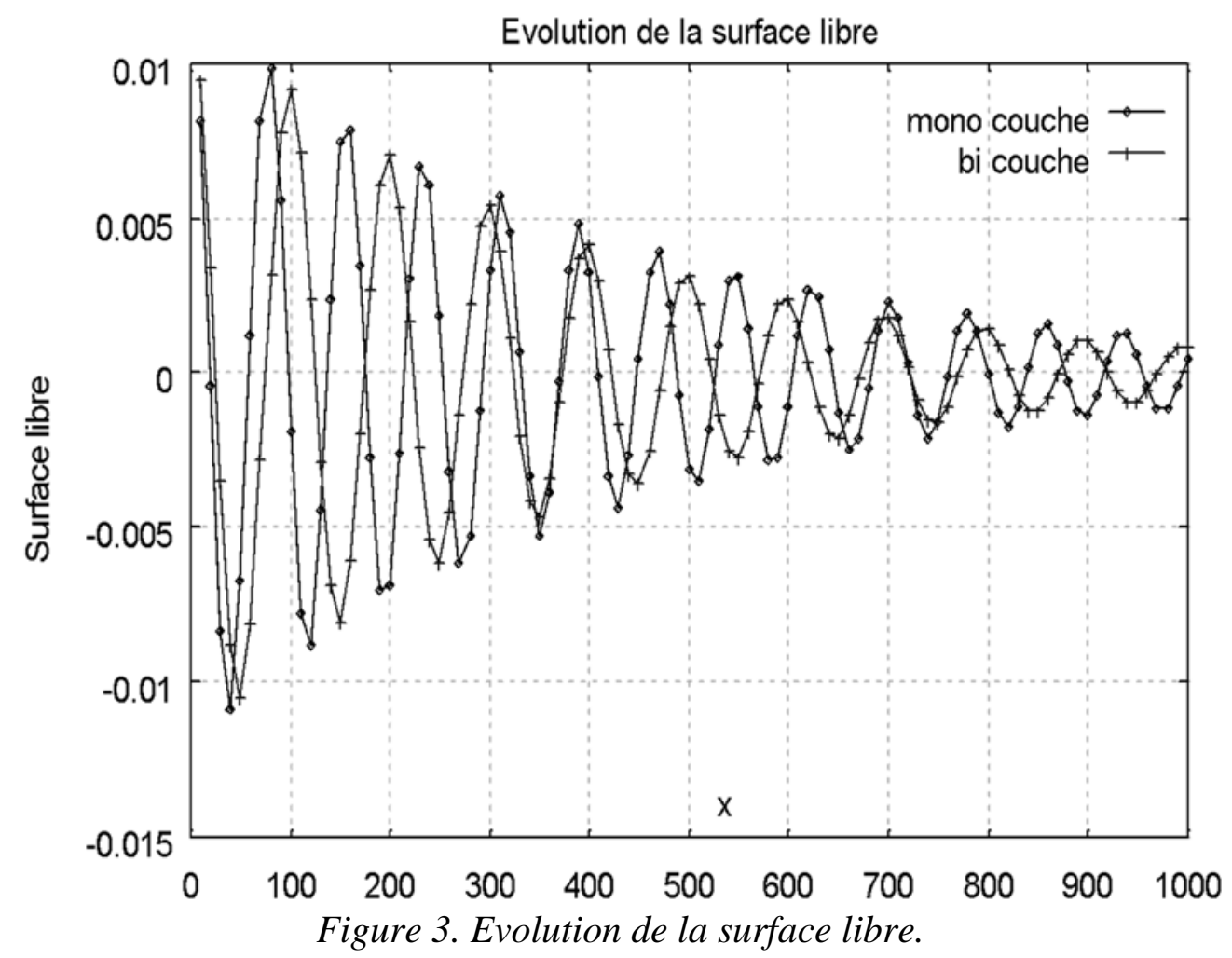




\section{XVèmes Journées Nationales Génie Côtier - Génie Civil \\ La Rochelle, 29 au 31 mai 2018}

L'évolution de la surface libre montre qu'un fond poreux composé de plusieurs couches de porosité différentes atténue la houle davantage par rapport à un milieu avec une seule couche poreuse. On a constaté que l'atténuation est tributaire des valeurs de la porosité. Il existe une analogie avec le fonctionnement des digues à talus.

La variation du coefficient d'amortissement en fonction de la fréquence n'est pas monotone. On observe qu'au-delà de la quatrième couche poreuse, il n'y a plus d'effet sur le coefficient d'amortissement et par conséquent sur l'atténuation de la houle.

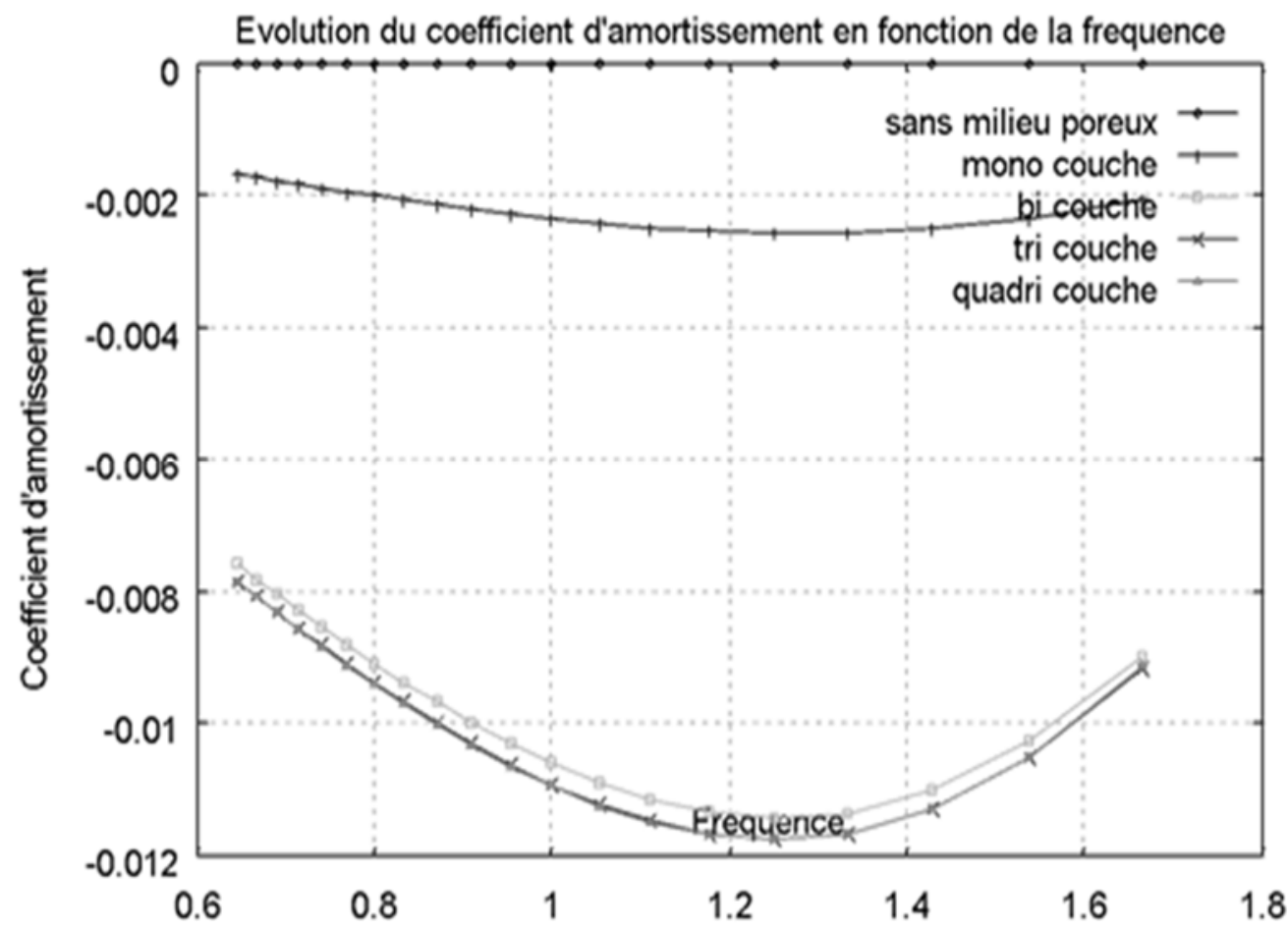

Figure 4. Evolution du coefficient d'amortissement en fonction de la fréquence.

\section{Conclusion}

Ce travail est validé expérimentalement dans le cas où le milieu poreux est constitué d'une seule couche (BROSSARD et al., 2004). Nous avons généralisé la procédure de calcul à un milieu constitué de plusieurs couches poreuses. Sous l'hypothèse de l'approximation des ondes longues nous avons fourni aussi une formulation avec une écriture simple. Cette écriture a permis d'introduire la notion de milieu équivalent.

En perspective, nous nos intéressons à des résolutions numériques pour conforter les solutions analytiques.

\section{Références bibliographiques}


Thème 1 - Hydrodynamique côtière

BROSSARD J., CHAGDALI M., REY V. (2004). Ecoulement engendré par la houle dans un milieu poreux. VIII èmes Journées Nationales Génie Côtier-Génie Civil, Compiègne, pp 47-54. https://doi.org/10.5150/jngcgc.2004.006-b

LEE J.F., LAN Y.J. (1995). A second - order solution of waves passing porous structures. Department of Hydraulics and Ocean Engineering, National Cheng Kung University, Tainan, Taiwan, R. O. C.

MADSEN P.A. (1983). Wave reflection from a vertical permeable wave absorber. Coastal Engineering, Vol. 7, pp 381-396. https://doi.org/10.1016/0378-3839(83)90005-4

SOLLITT C.K., CROSS R.H. (1972). Wave transmission through permeable breakwaters.13 ${ }^{\text {th }}$. Coastal Engineering Conf., vol.3, ASCE. Vancouver, pp.1827-1846. https://doi.org/10.1061/9780872620490.106

TEMPERVILLE A. (1985). Contribution à la théorie des ondes de gravité en eau peu profonde. Thèse d'Etat, Université Scientifique et médicale et Institut National Polytechnique de Grenoble. 